\title{
The future of traumatic brain injury research
}

\author{
Mark Wilson*, Parjam Zolfaghari, Collete Griffin, David Lockey, Christos Tolias, Vishi Verma \\ From London Trauma Conference 2013 \\ London, UK. 10-13 December 2013
}

\section{Introduction}

Traumatic Brain Injury (TBI) research is evolving. The quest for level-1 evidence through randomised prospective interventional trials, while useful to establish safety and efficacy, is changing to an era of big data observational studies. Comparative Effectiveness Research (CER) utilises the observation of differences between treatments and centres without strict inclusion/exclusion criteria or formal intervention [1]. In addition, the transfer of monitoring technologies into the pre-hospital field will enable research into earlier, more critical phases of brain injury. These two changes in TBI research direction should enable a better understanding of the spectrum of TBI diseases and foster a future of precision, personalised TBI management.

\section{Global epidemic of TBI}

Trauma is the commonest cause of death in under $45 \mathrm{~s}$ and TBI the commonest cause of this death [2]. Many survivors sustain considerable morbidity with lifelong effects for them, their family and society. In the US approximately 5.3 million live with disability following TBI. In Europe, this number is estimated to be 7.7 million [3] with $30-70 \%$ suffering on-going mental illness [4]. Despite this, TBI fails to attract research funding appreciated by more recognisable diseases such as cancer and heart disease.

\section{Problems with current TBI research}

\section{TBI classification}

TBI is traditionally classified as mild, moderate or severe based on initial Glasgow Coma Score (GCS) however, clinical experience tells us this is inaccurate. Patients with a GCS of 15 can die from an extradural and GCS 3 patients can be normal post-epileptic seizure. Approximately $25 \%$ of "mild" head injuries do not return to work and $84 \%$ have problems one year after injury, questioning how "mild" these injuries really are. Such classification is not appropriate for precision research. TBI is not one disease \footnotetext{
London's Air Ambulance and the Pan London Neurotrauma Group, London,
} UK

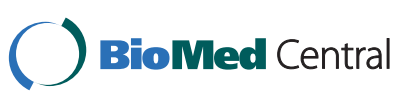

C 2014 Wilson et al; licensee BioMed Central Ltd This is an Open Access article distributed under the terms of the Creative Commons Attribution License (http://creativecommons.org/licenses/by/4.0), which permits unrestricted use, distribution, and reproduction in any medium, provided the original work is properly cited. The Creative Commons Public Domain Dedication waiver (http:// creativecommons.org/publicdomain/zero/1.0/) applies to the data made available in this article, unless otherwise stated. but a heterogenous collection (extradural/subdural, diffuse axonal etc) the outcome of which is determined by multiple factors such as injury location, physiology, extracranial injuries, and constitutional effects of the patient. Similarly, treatments are heterogeneous varying between centres and clinicians. Statistical adjustments (e.g. for extracranial injuries) are often inadequate and large observational studies are often retrospective. Whilst these may confirm common sense (e.g. treatment at a neurosciences centre improves outcome [5]; they also run the risk of generating self-fulfilling prophecy (e.g. the belief elderly patients do badly alters their care [6].

\section{Interventional trials}

"Level 1" evidence studies can be contrived for ethical or logistical purposes. Surgical intervention studies usually require surgeon equipoise before randomisation $[7,8]$. Do such results apply across the entire disease spectrum if only those in equipoise were entered? Neuroprotection drug administration within minutes of injury is difficult; hence most studies have a window of opportunity (e.g. 8 hours). Such studies have had a negative outcome $[9,10]$, however, neurons die within minutes of ischaemia and such logistical delays may create a type II error masking a real neuroprotective effect if given early.

Most interventional trials dichotomise outcomes but this can be clinically irrelevant and statistically inefficient. For example, a patient expected to be in vegetative state, owing to intervention, moves to a severely disabled state. Two novel approaches, sliding dichotomy and proportional odd models, overcome these inefficiencies. Sliding dichotomy requires patient stratification according to baseline risk and a point of dichotomy is identified [11]. For patients stratified in poor prognosis group, survival might be most relevant, whereas for those with a good prognosis any outcome worse than good recovery is undesirable. Robust prediction models are essential for such strategies [12]. Currently 2 models are available: CRASH and IMPACT while good at predicting death, fail to 
predict functional outcome. A large observational study of 3626 patients attempting to validate these risk prediction models, concluded that the IMPACT model was well calibrated for 6 months mortality but substantially under-predicted the risk of unfavorable outcome [12]. Large purpose built registries will refine such models.

Currently a number of fundamental neurotrauma principals (e.g. ICP monitoring) are being questioned [13]. An open mind is required to think again through these basic tenants.

\section{Timing of studies}

Most acute TBI research occurs on intensive care, a controlled safe environment. However, the time of greatest secondary injury is likely to be in the pre-hospital environment with hypoxia, hypotension, and expanding haematoma causing more neurological damage. It is this environment where intervention will have the greatest impact.

\section{The future of TBI research Big data}

A pragmatic approach to TBI research is required. A large European observational study of $5400 \mathrm{TBI}$ patients is about to start (http://www.center-tbi.eu). Using CER (across three strata of Emergency Department, Ward and Intensive Care admissions) and by studying treatment and outcome variations, best practice should be identified. Further collaboration with TrackTBI (the US TBI data portal) should harmonise TBI common data element collection.

\section{Pre-hospital research}

Many pre-hospital interventions (intubation, thoracotomy, blood administration) have been introduced in services like London's Air Ambulance. If pre-hospital neuropathology assessment could be achieved, then roadside personalised neurotrauma treatment may be possible. This has started in stroke medicine with dispatch of CT enabled ambulances to potential stroke patients [14]. On scene imaging enables rapid thrombolysis. Similarly earlier neuroprotective interventions may be possible with on scene TBI diagnosis.

\section{Pan-london neurotrauma group}

London is in a unique position for TBI data collection. The capital (daytime population $=12$ million) is serviced by four major trauma centres, each receiving from one ambulance service (London Ambulance Service) and one Air Ambulance (London's Air Ambulance). Additionally, all four receive severely injured patients from South East England via county air ambulances. This results in a population coverage of 16-18 million. The capital contains some of the top UK academic institutions that also have areas of specific interest within brain injury research. The pan-london neurotrauma group (http://www.londonneuro.com) will coordinate both clinical guideline development and academic collaboration. By creating an on-going prospective neurotrauma registry future interventional studies will be easier to instigate and run.

\section{Conclusion}

TBI is a major global problem, but new ways of studying it, both by collecting large quantities of data and by studying the early evolution of neurological injury offer the most exciting opportunities yet to better understand brain injury and provide disease targeted management plans.

Published: 7 July 2014

\section{References}

1. Maas AIR, et al: Re-orientation of clinical research in traumatic brain injury: report of an international workshop on comparative effectiveness research. Journal of neurotrauma 2012, 29(1):32-46.

2. WHO: 2nd Global Status Report on Road Safety. who.int 2011, Available at: http://www.who.int/violence_injury_prevention/road_safety_status/2013/ en/ [Accessed March 3, 2013].

3. Roozenbeek B, Maas AIR, Menon DK: Changing patterns in the epidemiology of traumatic brain injury. Nature reviews. Neurology 2013, 9(4):231-236.

4. Fazel S, Wolf A, Pillas D, Lichtenstein P, Langstrom N: Suicide, Fatal Injuries, and Other Causes of Premature Mortality in Patients With Traumatic Brain Injury. JAMA Psychiatry 2014, 71(3):326-333.

5. Patel HC, et al: Trends in head injury outcome from 1989 to 2003 and the effect of neurosurgical care: an observational study. Lancet 2005, 366(9496):1538-1544.

6. Kirkman MA, Jenks T, Bouamra O, Edwards A, Yates D, Wilson MH: Increased mortality associated with cerebral contusions following trauma in the elderly: bad patients or bad management? Journal of Neurotrauma 2013, 30(16):1385-1390.

7. Gregson BA, et al: Surgical trial in traumatic intracerebral hemorrhage (STITCH(Trauma)): study protocol for a randomized controlled trial. Trials 2012, 13(1):193-193.

8. Hutchinson PJP, et al: Decompressive craniectomy in traumatic brain injury: the randomized multicenter RESCUEicp study ([www.RESCUEicp. com]). Acta neurochirurgica. Supplement 2006, 96:17-20.

9. Edwards $P$, et al: Final results of MRC CRASH, a randomised placebocontrolled trial of intravenous corticosteroid in adults with head injuryoutcomes at 6 months. The Lancet 2005, 365(9475):1957-1959.

10. Hurlbert RJ: Methylprednisolone for acute spinal cord injury: an inappropriate standard of care. Journal of neurosurgery 2000, 93(1 Suppl):1-7.

11. Murray GD, et al: Design and analysis of phase III trials with ordered outcome scales: the concept of the sliding dichotomy. Journal of neurotrauma 2005, 22(5):511-517.

12. Harrison DA, et al: Risk Adjustment In Neurocritical care (RAIN)prospective validation of risk prediction models for adult patients with acute traumatic brain injury to use to evaluate the optimum location and comparative costs of neurocritical care: a cohort study. Health technology assessment (Winchester, England) 2013, 17(23):vii-350.

13. Chesnut RM, et al: A Trial of Intracranial-Pressure Monitoring in Traumatic Brain Injury. The New England journal of medicine 2012, 367(26):2471-2481.

14. Ebinger $M$, et al: Prehospital thrombolysis: a manual from Berlin. Journal of Visualized Experiments 2013, , 81: e50534-e50534.

doi:10.1186/1757-7241-22-S1-A7

Cite this article as: Wilson et al:: The future of traumatic brain injury research. Scandinavian Journal of Trauma, Resuscitation and Emergency Medicine 2014 22(Suppl 1):A7. 